\title{
Validation of the Academic Research Consortium for High Bleeding Risk (ARC-HBR) criteria in patients undergoing percutaneous coronary intervention and comparison with contemporary bleeding risk scores
}

Ueki, Yasushi ; Bär, Sarah ; Losdat, Sylvain ; Otsuka, Tatsuhiko ; Zanchin, Christian ; Zanchin, Thomas ; Gragnano, Felice ; Gargiulo, Giuseppe ; Siontis, George C M ; Praz, Fabien ; Lanz, Jonas ; Hunziker, Lukas ; Stortecky, Stefan ; Pilgrim, Thomas ; Heg, Dik ; Valgimigli, Marco ; Windecker, Stephan ; Räber, Lorenz

\begin{abstract}
AIMS The Academic Research Consortium for High Bleeding Risk (ARC-HBR) defined consensusbased criteria for patients at high bleeding risk (HBR) undergoing percutaneous coronary intervention (PCI). We aimed to validate the ARC-HBR criteria for the bleeding outcomes using a large cohort of patients undergoing PCI. METHODS AND RESULTS Between 2009 and 2016, patients undergoing PCI were prospectively included in the Bern PCI Registry. Patients were considered to be at HBR if at least one major criterion or two minor criteria were met. The primary endpoint was Bleeding Academic Research Consortium (BARC) 3 or 5 bleeding at one year; ischaemic outcomes were assessed using the device-oriented composite endpoints (DOCE) of cardiac death, target vessel myocardial infarction, and target lesion revascularisation. Among 12,121 patients, those at $\operatorname{HBR}(\mathrm{n}=4,781,39.4 \%)$ had an increased risk of BARC 3 or 5 bleeding $(6.4 \%$ vs $1.9 \%$; $<0.001)$ and DOCE $(12.5 \%$ vs $6.1 \%$; $<0.001)$ compared with those without HBR. The degree of risk and prognostic value were related to the risk factors composing the criteria. The ARC-HBR criteria had higher sensitivity than the PRECISE-DAPT score and the PARIS bleeding risk score $(63.8 \%, 53.1 \%, 31.9 \%)$, but lower specificity $(62.7 \%, 71.3 \%, 86.5 \%)$ for BARC 3 or 5 bleeding. CONCLUSIONS Patients at HBR defined by the ARC-HBR criteria had a higher risk of BARC 3 or 5 bleeding as well as DOCE. The bleeding risk was related to its individual components. The ARC-HBR criteria were more sensitive for identifying patients with future bleedings than other contemporary risk scores at the cost of specificity. ClinicalTrials.gov Identifier: NCT02241291 Visual summary. According to the ARC-HBR criteria, $40 \%$ of patients undergoing PCI were at HBR. Compared with patients without HBR, those at HBR had an increased risk of BARC 3 or 5 bleeding (6.4\% vs $1.9 \%$, $\mathrm{p}<0.001)$. There was a gradual risk increase for BARC 3 or 5 bleeding and DOCE as a function of the ARC-HBR score. BARC: Bleeding Academic Research Consortium; DOCE: device-oriented composite endpoints; HBR: high bleeding risk.
\end{abstract}

DOI: https://doi.org/10.4244/EIJ-D-20-00052

Posted at the Zurich Open Repository and Archive, University of Zurich

ZORA URL: https://doi.org/10.5167/uzh-191739

Journal Article

Published Version

Originally published at: 
Ueki, Yasushi; Bär, Sarah; Losdat, Sylvain; Otsuka, Tatsuhiko; Zanchin, Christian; Zanchin, Thomas; Gragnano, Felice; Gargiulo, Giuseppe; Siontis, George C M; Praz, Fabien; Lanz, Jonas; Hunziker, Lukas; Stortecky, Stefan; Pilgrim, Thomas; Heg, Dik; Valgimigli, Marco; Windecker, Stephan; Räber, Lorenz (2020). Validation of the Academic Research Consortium for High Bleeding Risk (ARC-HBR) criteria in patients undergoing percutaneous coronary intervention and comparison with contemporary bleeding risk scores. EuroIntervention, 16(5):371-379.

DOI: https://doi.org/10.4244/EIJ-D-20-00052 


\title{
Q
}

\section{Validation of the Academic Research Consortium for High Bleeding Risk (ARC-HBR) criteria in patients undergoing percutaneous coronary intervention and comparison with contemporary bleeding risk scores}

\author{
Eurolntervention 2020;16:371-379. DOI: 10.4244/EIJ-D-20-00052
}

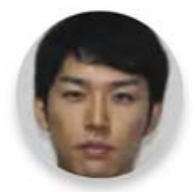

Yasushi Ueki', MD; Sarah Bär? , MD; Sylvain Losdat², PhD; Tatsuhiko Otsuka', MD; Christian Zanchin', MD; Thomas Zanchin', MD; Felice Gragnano', MD; Giuseppe Gargiulo', MD; George C.M. Siontis ${ }^{\top}$, MD, PhD; Fabien Praz', MD; Jonas Lanz', MD; Lukas Hunziker', MD; Stefan Stortecky', MD; Thomas Pilgrim? MD; Dik Heg², PhD; Marco Valgimigli', MD, PhD; Stephan Windecker?', MD; Lorenz Räber?', MD, PhD

7. Department of Cardiology, Bern University Hospital, Bern, Switzerland; 2. Institute of Social and Preventive Medicine and Clinical Trials Unit, University of Bern, Bern, Switzerland

Aims: The Academic Research Consortium for High Bleeding Risk (ARC-HBR) defined consensus-based criteria for patients at high bleeding risk (HBR) undergoing percutaneous coronary intervention (PCI). We aimed to validate the ARC-HBR criteria for the bleeding outcomes using a large cohort of patients undergoing $\mathrm{PCl}$.

Methods and results: Between 2009 and 2016, patients undergoing PCl were prospectively included in the Bern PCI Registry. Patients were considered to be at HBR if at least one major criterion or two minor criteria were met. The primary endpoint was Bleeding Academic Research Consortium (BARC) 3 or 5 bleeding at one year; ischaemic outcomes were assessed using the device-oriented composite endpoints (DOCE) of cardiac death, target vessel myocardial infarction, and target lesion revascularisation. Among 12,121 patients, those at HBR $(n=4,781,39.4 \%)$ had an increased risk of BARC 3 or 5 bleeding (6.4\% vs 1.9\%; $\mathrm{p}<0.001)$ and DOCE $(12.5 \%$ vs $6.1 \% ; \mathrm{p}<0.001)$ compared with those without HBR. The degree of risk and prognostic value were related to the risk factors composing the criteria. The ARC-HBR criteria had higher sensitivity than the PRECISE-DAPT score and the PARIS bleeding risk score (63.8\%, 53.1\%, 31.9\%), but lower specificity $(62.7 \%, 71.3 \%, 86.5 \%)$ for BARC 3 or 5 bleeding.

Conclusions: Patients at HBR defined by the ARC-HBR criteria had a higher risk of BARC 3 or 5 bleeding as well as DOCE. The bleeding risk was related to its individual components. The ARC-HBR criteria were more sensitive for identifying patients with future bleedings than other contemporary risk scores at the cost of specificity. ClinicalTrials.gov Identifier: NCT02241291 


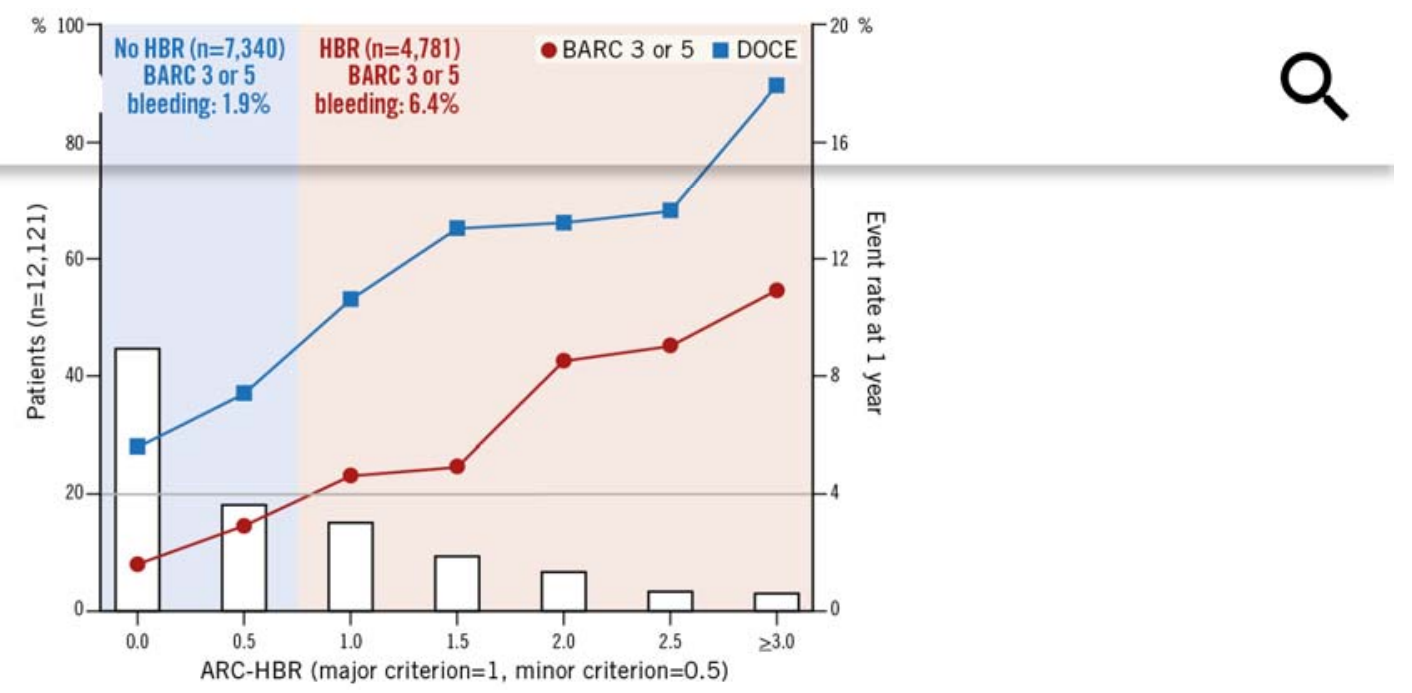

Visual summary. According to the ARC-HBR criteria, $40 \%$ of patients undergoing PCl were at $\mathrm{HBR}$. Compared with patients without HBR, those at HBR had an increased risk of BARC 3 or 5 bleeding $(6.4 \%$ vs $1.9 \%, p<0.001$ ). There was a gradual risk increase for BARC 3 or 5 bleeding and DOCE as a function of the ARC-HBR score. BARC: Bleeding Academic Research Consortium; DOCE: device-oriented composite endpoints; HBR: high bleeding risk.

\section{SIGN IN TO READ AND DOWNLOAD THE FULL ARTICLE}

\section{LOGIN}

\section{NO ACCOUNT YET? SIGN UP FOR FREE!}

\section{CREATE MY PCR ACCOUNT}

Join us for free and access thousands of articles from Eurolntervention, as well as presentations, videos, cases from PCRonline.com

acs/nste-acs bleeding coronary artery disease stable angina

\section{Read next article}

Clinical outcomes of calcified nodules detected by optical coherence tomography: a sub-analysis of the CLIMA study 
ke tua DEI.ATç, !SSUE

ruroIntervention

\section{Volume 16 Number 5}

$>$ VIEW CONTENTS

\section{TOOLBOX}

Print article

Citations

Ask for a reprint

Request permissions

\section{METRICS}

\section{Dimensions Badge}


10 Total citations
10 Recent citations
n/a Field Citation Ratio
n/a Relative Citation Ratio

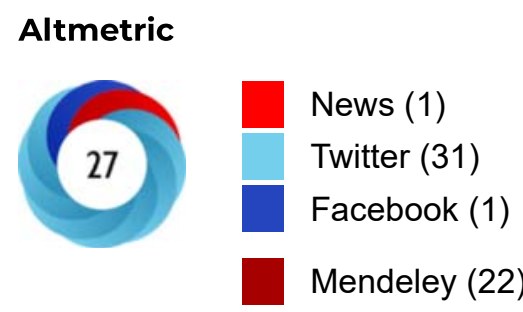

\section{POPULAR THIS MONTH}

An EAPCI Expert Consensus Document on Ischaemia with Non-Obstructive Coronary Arteries in Collaboration with European Society of Cardiology Working Group on Coronary Pathophysiology \& Microcirculation Endorsed by Coronary Vasomotor Disorders International Study Group.

Vijay Kunadian et al

Impact of the COVID-19 pandemic on interventional cardiology practice: results of the EAPCI survey

Marco Roffi et al

f 
2018 ESC/EACTS Guidelines on myocardial revascularization

Percutaneous recanalisation of chronic total occlusions: 2019 consensus document from the EuroCTO Club

Alfredo R. Galassi et al

\title{
LATEST NEWS
}

\author{
NEW ISSUE \\ Radiation protection, ticagrelor monotherapy, the COMPARE-ABSORB \\ trial, the BASILICA technique... \\ Severe calcification of the target leaflet \\ NOVEMBER 12, 2020
}

NEW

Impact of Intervention strategies after failed TMVR

NOVEMBER 12, 2020

EDITORIAL

ASA-free strategy in ACS

Usman Baber

NOVEMBER 12, 2020

\section{Popular Tavi-A, TAILOR-PCI, ATPCI, PORTICO-IDE and more} (Thanks to the support of Biotronik) 
Guidelines, consensus \& position papers

AUGUST 28, 2020

EDITORIAL

Bioresorbable scaffold déjà vu

Dean J. Kereiakes

NOVEMBER 4, 2020

JAA

\section{This Week's JAA's: Covid-19 in STEMI, 4 year outcomes of the EROSION study, Pulmonary ridge coverage after LAA occlusion...}

NOVEMBER 10, 2020

\section{CORONARY}

A randomized comparison of Coronary Stents according to Short or Prolonged durations of Dual Antiplatelet Therapy in patients with Acute Coronary Syndromes: a pre-specified analysis of the SMART-DATE trial Jang WJ et al

Predictors of Non-Stenting Strategy for Acute Coronary Syndrome Caused by Plaque Erosion: 4-Year Outcomes of the EROSION Study

He L et al

Dual antithrombotic therapy with dabigatran in patients with atrial fibrillation after percutaneous coronary intervention for ST elevation myocardial infarction: results from the randomised RE-DUAL PCI trial Zeymer $\mathrm{U}$ et al 


\section{VALVULAR DISEASE}

Fatal Acute Mesenteric Ischemia Following Transcatheter Aortic Valve Replacement del Val $D$ et al

Clinical Impact of Intervention Strategies after failed Transcatheter Mitral Valve Repair Alessandrini $\mathrm{H}$ et al

Percutaneous mitral valve leaflet repair: ongoing directions and future perspectives

Maisano F, Taramasso M

\section{HEART FAILURE}

The EURO SHOCK Trial: Design, Aims and Objectives Randomised comparison of Extra Corporeal Membrane Oxygenation (ECMO) delivered after acute-PCI plus standard of care versus standard of care alone after acute $\mathrm{PCl}$, in patients presenting with Acute Coronary syndrome and Cardiogenic Shock.

Banning $A$ et al

Percutaneous mitral valve leaflet repair: ongoing directions and future perspectives

Maisano F, Taramasso M

Deep Sedation versus General Anaesthesia for Transcatheter Mitral Valve Repair: An Individual-Patient Data Meta-analysis of Observational Studies.

Jobs A et al

\section{PERIPHERAL}

Multistage Strategy With Perfusion SPECT and CT Pulmonary Angiogram in Balloon Pulmonary Angioplasty for Totally Occluded Lesions in CTEPH

Hosokawa $\mathrm{K}$ et al

EAPCI Core Curriculum for Percutaneous Cardiovascular Interventions (2020): Committee for Education and Training European Association of Percutaneous Cardiovascular Interventions (EAPCI) A branch of the European Society of Cardiology

Van Belle E et al

Fistula between the right pulmonary artery and left atrium in a newborn: management and successful interventional treatment.

Michalak K et al

\section{HYPERTENSION}

EAPCI Core Curriculum for Percutaneous Cardiovascular Interventions (2020): Committee for Education and Training European Association of Percutaneous Cardiovascular Interventions (EAPCI) A branch of the European Society of Cardiology

Van Belle E et al

Early Results of the Revivent TC Procedure for Treatment of Left Ventricular Aneurysm and Heart Failure due to Ischemic Cardiomyopathy $\mathbf{A}$

Wang $Y$ et al

Pulmonary artery denervation using catheter-based ultrasonic energy $\mathbf{A}$ Rothman A et al 


\section{STROKE}

Impact of Pulmonary Ridge Coverage after Left Atrial Appendage Occlusion

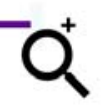

Freixa $X$ et al

Heterogeneity of debris captured by cerebral embolic protection filters during TAVI

Kroon $\mathrm{H}$ et al

First-in-human results of the OMEGA ${ }^{\mathrm{TM}}$ Left Atrial Appendage Occluder for Patients with Non-Valvular Atrial Fibrillation

Wilkins $\mathrm{B}$ et al

\section{About the journal}

Editorial team

Disclaimer

Privacy policy

\section{Readers}

Current issue

Archives

Subscribe

\section{Authors}

Submit your paper

Instructions

\section{Services}

Advertise

Reprints / ePrints

Rights and permissions

Textbooks 
The history of angioplasty

Q

Percutaneous cardiac interventions

Coronary stenosis

Follow us

Facebook

Twitter

Impact factor: 3.993

2019 Journal Citation Reports ${ }^{\circledR}$

Science Edition (Clarivate Analytics, 2020)

Online ISSN 1969-6213 - Print ISSN 1774-024X

(c) 2005-2020 Europa Group - All rights reserved 